PROCEEDINGS OF THE

AMERICAN MATHEMATICAL SOCIETY

Volume 125, Number 6, June 1997, Pages 1807-1809

S 0002-9939(97)03700-3

\title{
NONSTANDARD MODELS AND ANALYTIC EQUIVALENCE RELATIONS
}

\author{
SY D. FRIEDMAN AND BOBAN VELICKOVIC \\ (Communicated by Andreas R. Blass)
}

\begin{abstract}
We improve a result of Hjorth concerning the nature of thin analytic equivalence relations. The key lemma uses a weakly compact cardinal to construct certain nonstandard models, which Hjorth obtained using \#'s.
\end{abstract}

The purpose of this note is to improve the following result of Hjorth [2].

Theorem (Hjorth). Suppose that for every real $x, x^{\#}$ exists. Let $E$ be an analytic equivalence relation, $\Sigma_{1}^{1}$ in parameter $x_{0}$. Then either there exists a perfect set of pairwise $E$-inequivalent reals or every E-equivalence class has a representative in a set-generic extension of $L\left[x_{0}\right]$.

Hjorth's proof makes use of his analysis of nonstandard Ehrenfeucht-Mostowski models built from \#'s. Instead, we construct the necessary nonstandard models using infinitary model theory, assuming only the existence of weak compacts.

Theorem 1. Suppose that for every real $x$ there is a weakly compact cardinal in $L[x]$. Then the conclusion of Hjorth's Theorem still holds.

The main lemma is the following.

Lemma 2. Suppose that there is a weakly compact cardinal in $L[x], x$ a real. Then there is a countable nonstandard $\omega$-model $M_{x}$ of $Z F$ such that $x \in M_{x}$ and $L\left(M_{x}\right)=$ ( $L$ in the sense of $M_{x}$ ) has an isomorphic copy in a set-generic extension of $L\left[x_{0}\right]$, for any real $x_{0}$.

It is not known if the conclusion of Lemma 2 holds in $Z F C$ alone, for arbitrary $x$ (with $Z F$ replaced by an arbitrary finite subtheory).

Proof of Theorem 1 from Lemma 2 (as in Hjorth [2]). Suppose that $E$ is an analytic equivalence relation, $\Sigma_{1}^{1}$ in the parameter $x_{0}$, and choose an $x_{0}$-recursive tree $T$ on $\omega \times \omega \times \omega^{\omega}$ such that $x E y \longleftrightarrow T(x, y)$ has a branch. For each countable ordinal $\alpha$ we define $x E_{\alpha} y \longleftrightarrow \operatorname{rank}(T(x, y))$ is at least $\alpha$; then $E_{\alpha}$ is Borel in $\left(x_{0}, c\right)$ where $c$ is any real coding $\alpha$ and $E$ is the intersection of the $E_{\alpha}$ 's. We may assume that each $E_{\alpha}$ is an equivalence relation (see Theorem 1.4 of Hjorth [2]). A theorem of Harrington and Silver says that a $\Pi_{1}^{1}$-equivalence relation has a perfect set of pairwise inequivalent reals or each equivalence class has a representative constructible from the parameter defining the relation. As $E_{\alpha}$ is Borel in $\left(x_{0}, c\right)$ where $c$ is a

Received by the editors September 25, 1995 and, in revised form, November 20, 1995.

1991 Mathematics Subject Classification. Primary 04A15, 03C75.

Research supported by NSF Contract \# 92-05530.

(C)1997 American Mathematical Society 
real coding $\alpha$, and as we may assume that $E$ and hence each $E_{\alpha}$ has no perfect set of pairwise inequivalent reals, we know that each $E_{\alpha}$-equivalence class has a representative in $L\left[x_{0}, c\right]$ where $c$ is any real coding $\alpha$.

Now let $x$ be arbitrary and by Lemma 2 choose a countable nonstandard $\omega$ model $M_{x}$ of $Z F$ containing $\left(x_{0}, x\right)$ such that $L\left(M_{x}\right)$ has an isomorphic copy in a set-generic extension $N$ of $L\left[x_{0}\right]$. Let $a \in \operatorname{ORD}\left(M_{x}\right)$ be nonstandard and let $c$ be a code for $a$, generic over $M_{x}$; then by applying Harrington-Silver in $M_{x}[c]$ we conclude that there is $y$ in $L\left(M_{x}\right)\left[x_{0}, c\right]$ such that $y E_{a} x$. By choosing $c$ to be generic over $N$ as well we get that $y$ belongs to a set-generic extension of $L\left[x_{0}\right]$. Finally, $y E x$ since, if not, $y E_{\alpha} x$ would fail for some $\alpha$ admissible in $(y, x)$ and hence for some (standard) $\alpha<a$.

To prove Lemma 2 we discuss infinitary logic. Fix a real $x$ and assume $V=L[x]$. Let $\kappa$ be weakly compact and introduce the language $\mathcal{L}$ consisting of the formulas in the language of set theory with constants $\underline{a}$ for $a \in L_{\kappa}[x]$, closed under conjunctions and disjunctions of size less than $\kappa$ (however we allow a formula to have only finitely many free variables). Let $T$ be the theory of $\left\langle L_{\kappa}[x], a\right\rangle, a \in L_{\kappa}[x]$, in this language. An $n$-type is a set of formulas $\Gamma$ with free variables $v_{1} \ldots v_{n}$, and such a $\Gamma$ is consistent with $T$ if there is a model $M$ of $T$ and $m_{1} \ldots m_{n}$ in $M$ such that $M \models \varphi\left(m_{1} \ldots m_{n}\right)$ for each $\phi \in \Gamma$, where $M$ exists in a set-generic extension of $V=L[x] . \Gamma$ is complete if for every $\varphi\left(v_{1} \ldots v_{n}\right)$ either $\varphi$ or $\sim \varphi$ belongs to $\Phi$.

Now work in the Lévy collapse $L[x, c]$, where $c$ is a real coding $\kappa^{+}$of $L[x]$. Let $d_{1}, d_{2}, \ldots$ be $\omega$-many new constant symbols and for $D \subseteq\left\{d_{1}, d_{2}, \ldots\right\}$ let the language $\mathcal{L}_{D}$ be defined like $\mathcal{L}$ but with the new constant symbols from $D$. Define $T_{0}=T \subseteq T_{1} \subseteq \ldots$ and $D_{0}=\phi \subseteq D_{1} \subseteq D_{2} \subseteq \ldots$ inductively as follows: if $T_{n}, D_{n}$ have been defined select a complete $k$-type $\Gamma_{n}(\vec{v})$ in $L[x]$ consistent with $T_{n}$, choose $D_{n} \subseteq D_{n+1}$ so that $\operatorname{card}\left(D_{n+1}-D_{n}\right)=k$, and let $T_{n+1}=T_{n} \cup \Gamma_{n}(\vec{d})$, where $\vec{d}$ enumerates $D_{n+1}-D_{n}$. This can be done in such a way that $\bigcup_{n} T_{n}=T^{*}$ is $L[x]$-saturated: if $\Gamma(\vec{v}, \vec{w})$ is an $L[x]$-type, $\vec{d}$ a finite sequence from $D$, and $\Gamma(\vec{d}, \vec{w})$ consistent with $T^{*}$, then $T^{*}$ includes $\Gamma(\vec{d}, \vec{e})$ for some $\vec{e}$. And note that each $T_{n}$ belongs to $L[x]$ (though of course $T^{*}$ itself makes use of the Lévy collapse $c$ ).

Let $M_{x}$ be the model determined by $T^{*}$, whose universe consists of (equivalence classes of) the constants $d_{n}, n \in \omega$. Note that a set in $L[x]$ of sentences in some $\mathcal{L}_{D}$ is consistent iff each subset of $L[x]$-cardinality $<\kappa$ is, by $\Pi_{1}^{1}$-reflection and the equivalence of consistency with existence of a model after performing a Lévy collapse of $\kappa$. An easy consequence is that $M_{x}$ is nonstandard with standard ordinal $\kappa$.

Now consider $L\left(M_{x}\right)$ : every $n$-type in the language $\mathcal{L}_{0}=$ (same as $\mathcal{L}$ but restricted to $\left.L_{\kappa}\right)$ that is realized in $L\left(M_{x}\right)$ belongs to $L$, as each of its initial segments (obtained by restricting to some $L_{\alpha}, \alpha<\kappa$ ) belongs to $L$ and $\kappa$ is weakly compact. Also, just as $M_{x}$ is saturated for types in $L[x], L\left(M_{x}\right)$ is saturated for types in $L$, since again by weak compactness any $\mathcal{L}_{0}$-type in $L$ consistent with $T$ can be extended to a complete $\mathcal{L}$-type consistent with $T$ in $L[x]$.

Now it is clear that $L\left(M_{x}\right)$ has an isomorphic copy in $L[c]$ : using $c$ we can do the same construction as we did above in $L[x, c]$, obtaining $M_{0}$, a model that is saturated for $\mathcal{L}_{0}$-types in $L$ and realizing only types in $L$. Now construct an isomorphism via a back and forth argument in $\omega$ steps between $M_{0}$ and $L\left(M_{x}\right)$.

Finally, note that by absoluteness the desired model $M_{x}$ exists not only in $L[x, c]$ but in $L[x]$. 
Remark. Lemma 2 can also be used to establish the following improvement of the Glimm-Effros style dichotomy theorem of Hjorth and Kechris [3]: Let $E$ be a $\Sigma_{1}^{1}$ equivalence relation. Assume that for every real $x$ there is a weakly compact cardinal in $L[x]$. Then either $E_{0}$ is continuously reducible to $E$, or $E$ is reducible to $2^{<\omega_{1}}$ by a function $\Delta_{2}^{1}$ in the codes.

\section{REFERENCES}

[1] L. Harrington and S. Shelah, Counting equivalence classes for co- $\kappa$-Souslin equivalence relations, Logic Colloquium '80, North-Holland, 1982, pp. 147-152. MR 84c:03088

[2] G. Hjorth, Thin analytic equivalence relations and effective decompositions, J. Symbolic Logic. 58 (1993), 1153-1164. MR 95c:03119

[3] G. Hjorth and A. S. Kechris, Analytic equivalence relations and Ulm-type classifications, J. Symbolic Logic 60 (1995), 1273-1300. CMP 96:06

[4] J. H. Silver, Counting the number of equivalence classes for Borel and coanalytic equivalence relations, Ann. Math. Logic 18 (1980), 1-28. MR 81d:03051

Department of Mathematics, M.I.T., Cambridge, Massachusetts 02139

E-mail address: sdf@math.mit.edu

Equipe de Logique, University of Paris 7, 2 Place Jussieu, 75251 Paris Cedex 05, FRANCE

E-mail address: boban@logique.jussieu.fr 